\title{
Método para estimativa do IAF de árvores isoladas ou de plantações com dossel fechado
}

\author{
Maurício A. Coelho Filho', Nilson A. Villa-Nova ${ }^{2}$, Luiz R. Angelocci' ${ }^{2}$, \\ Fábio R. Marin ${ }^{3}$ \& Ciro A. Righi ${ }^{2}$
}

\begin{abstract}
RESUMO
Objetivou-se, com o presente trabalho, descrever e testar um método simplificado de baixo custo (IAFLUX) para estimativa do índice de área foliar (IAF) em comparação com um equipamento comercial (LAI2000, Li-Cor"). Ambos foram avaliados em um seringal e em árvores de lima ácida "Tahiti"; posteriormente, os dois métodos foram comparados com medidas destrutivas de área foliar realizadas em uma árvore adulta de lima ácida 'Tahiti'; notou-se, então, que os desvios dos resultados pelo IAF-LUX em relação aos do LAI-2000 foram no máximo de $7,4 \%$ na seringueira e de 3,6\% em lima ácida "Tahiti". A estimativa da área foliar total utilizando-se o LAI 2000, mostrou boa concordância com as medidas diretas; resultados semelhantes foram obtidos com o IAF-LUX (5\% de erro). Para as condições em que foi desenvolvido o trabalho os métodos com base em medida de radiação apresentaram boa precisão na estimativa da área foliar total das árvores de lima ácida, ficando patente que este método pode substituir vantajosamente as outras técnicas usadas, em comparação pelo baixo custo do equipamento, pela facilidade de operação no campo e análise dos dados.
\end{abstract}

Palavras-chave: LAI-2000, luxímetro, citros, Hevea spp

\section{Method to estimate the LAI for isolated trees and continuous canopy crops}

\begin{abstract}
This paper aimed to describe and evaluate a low-cost simplified method (IAF-LUX method) for leaf area index (LAI) estimation, in comparison with well-tested commercial equipment (LAI-2000, $\mathrm{Li}^{-\mathrm{Cor}^{\circledR}}{ }^{\circledR}$, being both applied for a rubber tree plantation and for a 'Tahiti' acid lime orchard. Later, the results of the both methods were also compared with destructive measurements of leaf area carried out in other 'Tahiti' acid lime adult tree. Considering LAI-2000 as reference, the proposed IAF-LUX method showed deviations of $7.4 \%$ for rubber tree and $3.6 \%$ for acid lime 'Tahiti'. Comparing the IAF-LUX with destructive measurements, a deviation of $5.0 \%$ was observed in acid lime, while LAI-2000 showed deviation of $7.0 \%$. Based on these results, it is concluded that the IAF-LUX method may be used for determining leaf area in the two species, replacing advantageously the other destructive or non-destructive techniques because it is of low cost, easily operated and non-destructive procedure.
\end{abstract}

Key words: Lai 2000, luximeter, citrus, Hevea spp

\footnotetext{
Embrapa Mandioca e Fruticultura Tropical, Rua Embrapa, s/n, CP 07, CEP 44380 000, Cruz das Almas, BA. Bolsista do CNPq. E-mail: macoelho@cnpmf.embrapa.br

2ESALQ/USP, CP 9, CEP 13418-900, Piracicaba, SP. Bolsista do CNPq. E-mail: navnova@esalq.usp.br; Irangelo@esalq.usp.br; ciro@esalq.usp.br Embrapa Informática Agropecuária, Av. André Tosello, 209 - Barão Geraldo, CP 6041, CEP 13083-886, Campinas, SP. E-mail: marin@cnptia.embrapa.br
} 


\section{INTRODUÇÃO}

O conhecimento da estrutura de um dossel vegetativo é importante para o maior entendimento das repostas vegetais a estímulos ambientais pois as inter-relações existentes entre vegetal e ambiente são governadas por essa variável (Norman \& Campbell, 1989). Aárea foliar e a variável derivada "índice de área foliar"(IAF) são determinantes da produtividade (Righi \& Bernardes, 2008) e do consumo de água da vegetação (Macfarlane et al., 2000), de vez que participam na interceptação da radiação solar e nas trocas energéticas e gasosas, afetando a transpiração (Angelocci \& Valancogne, 1993; Coelho Filho et al., 2004; 2007; Righi et al., 2008), a evaporação da água no solo (Allen et al., 1998) e a fotossíntese (Hollinger, 1992).

$\mathrm{Na}$ determinação da área foliar (AF) podem ser usados métodos diretos e indiretos, destrutivos ou não. Os métodos diretos estão relacionados às medidas tomadas diretamente na planta, sendo muito importantes para estimativa adequada e amostragem representativa, que será maior ou menor em função do tamanho e do tipo da cultura. Os métodos diretos apresentam maior precisão quando bem aplicados mas aumentam progressivamente o tempo de dedicação da análise à medida em que aumentam a complexidade do dossel e a conformação do plantio.Várias dificuldades ocorrem nessa determinação em espécies arbóreas, principalmente em função do porte e do número de folhas de árvores adultas. Dificuldades adicionais podem ser observadas nos trabalhos realizados em condições de sistemas agroflorestais ou florestas (Macfarlane et al., 2000; Welles \& Cohen, 1996), quando a cobertura é heterogênea, dificultando a amostragem.

Um grupo de métodos indiretos se baseia em medidas da transmissão da luz direta e/ou difusa no dossel vegetativo, com possibilidade de armazenamento dos dados em memória eletrônica e posterior utilização com tratamento informatizado. Tais métodos apresentam a vantagem de não ser destrutivos e de permitirem maior frequência de leituras pela rapidez de mensuração, utilizando apenas a informação da fração de radiação transmitida que não sofreu atenuação pela estrutura do dossel vegetativo. Há instrumentos comercializados que usam este princípio e com os quais se tem obtido resultados satisfatórios, desde que adequadamente operados para minimizar erros ou realizar correções e ajustes, dependendo do tipo e da idade das plantas. Por exemplo, cita-se seu uso em gramíneas de pequeno porte (Stroppiana et al., 2006; Sbrissia \& Silva, 2008). O uso de métodos de simples medida e análise é interessante por permitir maior amostragem, obtendo-se valores médios comparáveis no tempo, sendo de utilidade em estudos de variabilidade temporal de IAF (Chason et al., 1991; Xavier et al., 2002), de interação fertilidade do solo e IAF (Smethurs et al., 2002), de dinâmica de dossel (Paula \& Lemos Filho, 2001) e de produção de biomassa (Linhares et al., 2000) entre outros.

Dentre os muitos aparelhos comercializados que utilizam modelos que envolvem a fração transmitida de radiação se destacam os sensores quânticos, tais como o "LAI-2000" (LiCor $^{\circledR}$ ) (Norman \& Campbell, 1989; Welles, 1990; Weles \& Norman, 1991), o "demon" (Lang et al., 1985), o "ceptômetro" (Welles \& Cohen, 1996), os sensores utilizados em fotografias hemisféricas (Rich, 1990; Leblanc et al., 2005; Zhang et al., 2005; Demarez et al., 2008) e os sensores de respostas espectrais (Schlerf et al., 2005; Fuentes et al., 2008; Brantley et al., 2011). As potenciais fontes de erro dos métodos que usam o princípio da transmissão de luz pelo dossel, estão associadas à interceptação da radiação por ramos e troncos, com superestimativa de resultados principalmente em condições de baixo IAF, e à aglomeração de folhas, que fere a pressuposição de distribuição aleatória das folhas no plano de projeção exigida nesse tipo de abordagem (Welles, 1990). Com o uso dessas técnicas foram observadas subestimativas do IAF em eucalipto (Norman \& Campbell, 1989; Macfarlane et al., 2000), em florestas coníferas (Fassnacht et al., 1994; Stenberg, 1996), em pinus (Lang et al., 1985), em girassol (Norman \& Campbell, 1989), sobremaneira quando o IAF é elevado. Referidas diferenças podem ser corrigidas por coeficientes ou modelos envolvendo parâmetros vegetativos (Kucharik et al., 1998).

As fotografias hemisféricas, tal como no trabalho com seringueira, por Leong et al. (1982), devem ser utilizadas tomando-se as devidas precauções com as prováveis fontes de erro, relacionadas à má exposição das fotografias que afeta o contraste entre as folhas e o céu, a deformação causada pela projeção da imagem hemisférica em um plano e o espalhamento e difração da luz incidente pelas folhas (Bignami \& Rossini, 1996). Monte et al. (2007) usaram fotografias digitais comuns nas estimativas de índice de cobertura vegetal, correlacionando os IAF estimados com o uso do LAI-2000 e encontraram correlações significativas entre as duas estimativas. Macfarlane et al. (2007) também encontraram boas estimativas com ambos os métodos, analisando floresta de eucalipto.

Dentre os equipamentos existentes para obtenção indireta do IAF o analisador de dossel LAI-2000 (Li-Cor $\left.{ }^{\circledR}\right)$ se destaca por sua praticidade e rapidez na obtenção dos dados (Welles, 1990), além de fornecer estimativa confiável do IAF (Norman \& Campbell, 1989). O LAI-2000 tem sido amplamente empregado em estudos de estimativa de AF e do índice de área foliar (IAF) em diferentes culturas (Sommer \& Lang, 1994; Villalobos et al., 1995; Herbert \& Fownes, 1997; Cutini et al., 1998; Barclay \& Trofmow, 2000; Xavier et al., 2002; Xavier \& Vettorazzi, 2003; Monte et al., 2007; Arias et al., 2007; Lara et al., 2011; Silva et al., 2011; Majasalmi et. al., 2012). Este medidor é equipado com uma lente hemisférica tipo "olho de peixe", subdividida em cinco bandas angulares de visada com relação ao zênite $(7,23$, 38,53 e $68^{\circ}$ ). Desta maneira, a fração de luz que transpõe o dossel vegetativo (transmitância) nos diferentes ângulos é armazenada e utilizada no cálculo do IAF; um filtro óptico restringe as medidas de transmitância à faixa de luz abaixo de 490nm, minimizando os erros da reflexão pelas folhas.

Apesar de suas características vantajosas, o LAI-2000 tem custo relativamente elevado e a possibilidade de simplificar a técnica e minimizar tal custo é bastante interessante. Com isto em mente, o presente trabalho tem por objetivo apresentar e validar uma metodologia simplificada para determinação da área foliar de plantas adultas de lima ácida 'Tahiti' e do índice de área foliar (IAF) em maciço de seringueira, utilizando um luxímetro de baixo custo. 


\section{Material e MÉTODOS}

O trabalho foi desenvolvido na Escola Superior de Agricultura Luiz de Queiroz (ESALQ/USP), Piracicaba, SP, em duas condições:

Cultura com cobertura contínua: sob condições de cobertura total e uniforme do terreno pelo dossel, foram realizadas algumas medidas de índice de área foliar (IAF - $\mathrm{m}^{2}$ de folha $\mathrm{m}^{-2}$ de solo) em um maciço de seringueira composto de plantas adultas;

Árvores isoladas: foram avaliadas cinco plantas de lima ácida 'Tahiti' em um pomar de oito anos de idade. Os valores de índice de área foliar tendo por base a área de projeção vertical da copa no solo (DLIAF - $\mathrm{m}^{2}$ de folha $/ \mathrm{m}^{2}$ de projeção de copa) e área foliar $\mathrm{AF}\left(\mathrm{m}^{2}\right)$ calculados com o método proposto denominado, a partir deste ponto, como IAF-LUX, foram comparados com os valores obtidos pelo equipamento "LAI2000" (Li-Cor, 1992). Na utilização do equipamento comercial foram seguidas todas as recomendações de operação e de cálculo do fabricante. Para duas plantas avaliadas foram realizadas medidas diretas comparativas enquanto em outras quatro plantas apenas as metodologias indiretas foram empregadas. Os métodos diretos utilizados como referência envolveram medidas de dimensões foliares e contagem de folhas, além de determinação de massa seca.

São detalhados, a seguir, os procedimentos adotados em cada um dos métodos de estimativas (diretos e indiretos) utilizados no presente trabalho.

\section{Estimativas com base em medidas de dimensões e número de folhas}

A área foliar foi medida utilizando-se três diferentes métodos diretos:

1. Relação entre massa seca foliar e área foliar (MSAF): as folhas foram removidas, colocadas em sacos de papel e levadas ao laboratório. A massa seca (MS) das folhas em cada saco foi obtida em balança com sensibilidade $0,0001 \mathrm{~g}$ e convertida em área foliar por uma equação de regressão linear (MS x AF). A área foliar de algumas amostras (sacos) foi determinada diretamente pelo medidor foliar modelo LI $3000 \mathrm{~A}\left(\mathrm{Li}_{-} \mathrm{Cor}^{\circledR}\right)$, sendo que o número de folhas utilizadas para o estabelecimento dessa regressão correspondeu a $2 \%$ do total das folhas de uma árvore com 90.483 folhas.

2. Contagem de folhas $(\mathrm{CF})$ : as folhas foram removidas das árvores em vários dias subsequentes em função da sua grande quantidade. Ao final de cada dia de coleta as folhas eram contadas e em seguida separadas aleatoriamente amostras representativas para medida direta de área foliar. Obteve-se a área foliar total da planta ao se multiplicar o número total de folhas contadas pela área foliar média das folhas removidas.

3. Dimensões lineares (DL): o aparelho LI 3000A armazena os valores de medidas lineares da folha avaliada, ou seja, o comprimento (C) e a maior largura (L). Esses valores foram utilizados para estabelecer uma relação linear do tipo $\mathrm{AF}=$ $\mathrm{K}_{*}\left(\mathrm{C}_{*} \mathrm{~L}\right)$, que foi utilizada na estimativa da área foliar média das folhas. Tal como realizado no item "ii”, o valor da área foliar total foi obtido multiplicando-se a área foliar média das folhas pelo seu número total.

\section{Estimativas com base em técnicas de transmissão de luz}

Duas técnicas de medidas de IAF foram utilizadas com base na transmitância de radiação no dossel vegetativo;a primeira, por meio de sensor comercial (LAI-2000) e a segunda com um luxímetro digital, a partir de modificações na base teórica utilizada na primeira dessas técnicas.

\section{Determinação com o sensor LAI-2000}

Tomaram-se os cuidados necessários com relação às medidas feitas, sendo realizada uma medida sem a interferência das plantas $\left(\mathrm{I}_{0}\right)$ e outra sob sua interferência, abaixo do dossel (I) em pontos escolhidos criteriosamente segundo as recomendações do fabricante (Li-Cor, 1992) e as características das plantas avaliadas cobrindo, completa ou parcialmente (plantas isoladas) o solo. Como a nova técnica introduzida neste trabalho é uma modificação da usada no LAI-2000 apresenta-se também, a seguir, o tratamento teórico usado.

Os cálculos foram realizados por meio do programa C2000, desenvolvido pelo fabricante para análise dos dados. $\mathrm{O}$ programa calcula, primeiro, a densidade foliar $\mu$ (Eq. 1) e, posteriormente, a área foliar total (AFT) advinda da informação referente ao volume da copa (Eq. 2).

A partir dos resultados obtidos nos cinco ângulos zenitais fixados pelo aparelho, a equação seguinte foi utilizada nos cálculos da área foliar:

$$
\text { L ou } \mu=2 \sum_{1}^{5} \frac{-\ln \left(\mathrm{T}_{\mathrm{i}}\right)}{\mathrm{S}_{\mathrm{i}}} \mathrm{W}_{\mathrm{i}}
$$

em que:

$\mathrm{T}_{\mathrm{i}}$ - frações transmitidas em cada ângulo zenital $\mathrm{i}$

$\mathrm{W}_{\mathrm{i}}$ - valores de sin $\alpha \mathrm{d} \alpha$ para os quatro ângulos de visada do instrumento

$\mathrm{S}_{\mathrm{i}} \quad$ - valores de $1 / \cos \alpha$ para cada ângulo

Em culturas cobrindo completamente o terreno ou em renque, toma-se o cuidado de fazer leituras em diagonal às linhas de plantio visando-se obter valores representativos de transmitância e, assim, a solução da Eq. 1 corresponderá ao IAF. Para árvores ou plantas isoladas cobrindo uma parcela $\varphi$ $\left(\mathrm{m}^{2}\right)$ do terreno, a solução corresponderá ao valor da densidade foliar $\mu\left(\mathrm{m}^{2} \mathrm{~m}^{-3}\right)$. Para este último caso a estimativa do volume $\phi$ $\left(\mathrm{m}^{3}\right)$ ocupado pelo dossel é bastante significativo haja vista que afetará o resultado final ao se calcular a área foliar total $(\mathrm{AF})$ :

$$
\mathrm{AF}=\mu . \phi
$$

Em árvores isoladas os valores de $\mathrm{S}_{\mathrm{i}}$ variam com o formato do dossel motivo pelo qual os cálculos deverão ser realizados utilizando-se os valores corretos para cada planta avaliada. Os valores médios de Si foram calculados utilizando-se o programa C2000 (Li-Cor, 1992) e, para tal propósito, são necessárias as coordenadas (xy) que representam o perfil de copa analisado. O número de subdivisões da copa da árvore utilizadas para se chegar a um valor confiável de densidade foliar (Eq. 1) será 
tanto maior quanto maior for a irregularidade geométrica da árvore.

Apesar das árvores analisadas apresentarem, aparentemente, perfis geométricos aproximadamente regulares, três plantas analisadas foram divididas em quatro quadrantes cada uma, fornecendo-se ao programa o formato da copa para cada quadrante analisado, além do posicionamento espacial do sensor em cada quadrante, utilizando-se coordenadas (xy). As leituras acima e abaixo do dossel foram realizadas com uma abertura de $90^{\circ}$ amostrando $1 / 4$ da radiação que chega ao sensor, limitando a visada ao quadrante avaliado ao se bloquear a radiação proveniente dos outros três setores. Cada medida realizada pelo aparelho foi correlacionada com valores encontrados nos métodos diretos estudados que também foram avaliados por quadrantes. Em duas outras plantas as medidas foram realizadas apenas em dois setores na direção da linha de plantio (Figura 1A), utilizando-se abertura de $180^{\circ}$ e amostrando $1 / 2$ da radiação que chega no sensor (Figura 1A).

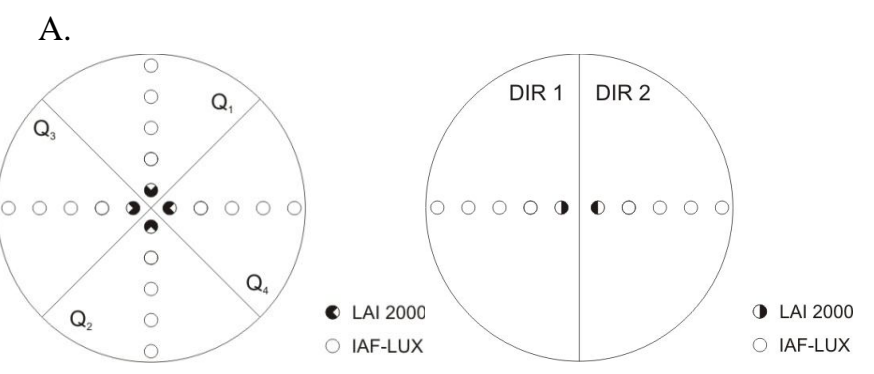

B.

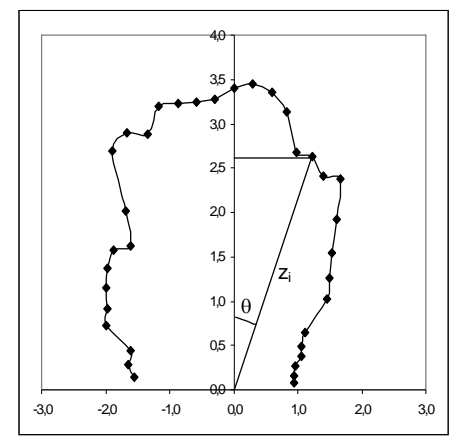

Figura 1. Esquema utilizado na coleta dos dados dos métodos indiretos testados (LAI-200 e IAF-LUX) em plantas de lima ácida 'Tahiti' $(\mathrm{A})$; representação esquemática da divisão da copa $\left(\theta=5^{\circ}\right)$ para obtenção do valor de $z$ (B)

O posicionamento do aparelho para realização das medidas foi escolhido criteriosamente com a finalidade de se minimizar erros relacionados à presença de ramos grossos na linha de visada. A possível contribuição do tronco da planta foi eliminada da análise em função da utilização da abertura de 90 e $180^{\circ}$ da placa de captação da radiação e as medidas foram realizadas ao entardecer, período do dia em que predomina a fração de radiação difusa, minimizando-se os erros relacionados à incidência de radiação direta no sensor (Welles \& Norman, 1991).

\section{Medidas com luxímetro: Descrição do método (IAF-LUX)}

Para o segundo método utilizado (IAF-LUX), os fluxos luminosos foram medidos com um luxímetro convencional acoplado a um filtro azul, que permitia a leitura da radiação solar somente entre os comprimentos de onda entre 400 e 490 $\mathrm{nm}$ (na região do azul), de modo que apenas esta fração de comprimento de onda difundida fosse medida pelo equipamento não se computando as reflectâncias nem as transmitâncias complementares propagadas ao longo do dossel; da mesma forma usada com o LAI-2000, as medidas foram realizadas ao entardecer com predominância de radiação difusa no feixe radiativo.

De acordo com Welles et al. (1990), quando um feixe de radiação incide em um dossel ocorre a probabilidade de ele ser interceptado pela vegetação, probabilidade esta proporcional à densidade foliar, à extensão do dossel e à orientação foliar. Se as folhas do dossel forem distribuídas randomicamente e forem pequenas comparadas com as dimensões do dossel, então o feixe de radiação numa direção relativa ao ângulo zenital $(\alpha)$ e azimutal $(\theta)$ tem a probabilidade de não interceptação descrita pela Eq. 3:

$$
\mathrm{T}(\alpha, \theta)=\exp [-\mathrm{G}(\alpha, \theta) \mu \mathrm{S}(\alpha, \theta)]
$$

em que $\mathrm{G}(\alpha, \theta)$ é o coeficiente de extinção definido como a fração de área foliar nas direções $(\alpha, \theta), \mu$ é a densidade foliar $\left(\mathrm{m}^{2}\right.$ de folha por $\mathrm{m}^{3}$ do dossel vegetativo) e $\mathrm{S}(\alpha, \theta)$ é a distância em que o feixe atravessa o dossel (m).

Para culturas que cobrem completamente o terreno, como no seringal, a densidade foliar é relacionada com o índice de área foliar (IAF) mediante a altura do dossel vegetativo z; a trajetória $\mathrm{S}$ é relacionada com a espessura do dossel no ângulo a da seguinte da maneira:

$$
\begin{gathered}
\mathrm{IAF}=\mu \mathrm{z} \\
\mathrm{S}(\alpha)=\frac{\mathrm{z}}{\cos \alpha}
\end{gathered}
$$

Fazendo-se a substituição dessa relação na Eq. 1, pode-se escrever:

$$
\mathrm{IAF}=\frac{-\ln (\mathrm{T}(\alpha))}{\mathrm{G}(\alpha)} \cos \alpha
$$

No método admite-se que o valor do coeficiente de extinção possa ser estimado por:

$$
G(\alpha)=1-T(\alpha)
$$

Como o luxímetro não possui ângulos de visadas hemisféricas definidas, desconsiderou-se este fator $\left(\cos 0^{\circ}=1\right)$ e a equação final para cálculo pode ser assim representada ao substituir a Eq. 7 em 6:

$$
\mathrm{IAF}=-\frac{\operatorname{LnT}(\alpha)}{1-\mathrm{T}(\alpha)}
$$


ou

$$
\mathrm{IAF}=-\frac{\operatorname{Ln}\left(\frac{1}{\mathrm{n}} \sum \frac{\mathrm{I}}{\mathrm{I}_{\mathrm{o}}}\right)}{1-\frac{1}{\mathrm{n}} \sum \frac{\mathrm{I}}{\mathrm{I}_{\mathrm{o}}}}
$$

sendo $I_{\mathrm{o}}$ e I as medidas realizadas sem a interferência do dossel e abaixo dele, respectivamente.

Existem duas situações típicas de cobertura vegetal que devem ser analisadas separadamente: a primeira é quando o dossel vegetativo cobre completamente o terreno, condições em que os valores de $\mu$ e $S$ são aproximadamente constantes e a Eq. (8) é suficiente para determinação do IAF com aplicação direta nos estudos com seringueira. A segunda situação é quando o dossel cobre parcialmente o terreno (árvores isoladas), caso em que o sistema de cálculo deve ser de tal forma que o DLIAF calculado seja representativo do dossel avaliado. Desta maneira, a Eq. 8 deverá ser corrigida por uma altura média equivalente da copa $(\overline{\mathrm{z}})$, considerando-se, daí, a aproximação de que a árvore avaliada possui densidades foliares homogêneas, quando então o IAF calculado passa a ser equivalente numericamente à densidade foliar e a $\mathrm{AF}$ é calculada pela Eq. 9 .

$$
\mathrm{AF}=-\frac{\operatorname{LnT}(\alpha)}{1-\mathrm{T}(\alpha)} \overline{\mathrm{z}} \varphi
$$

O parâmetro $\varphi$ (área de solo correspondente à projeção da copa da árvore $-\mathrm{m}^{2}$ ) foi inserido na Eq. 9 para transformar o valor de DLIAF calculado (adimensional $\mathrm{m}^{2} \mathrm{~m}^{-2}$ ) em área foliar $\left(\mathrm{m}^{2}\right)$. A altura equivalente é obtida analisando-se a silhueta da árvore em duas dimensões (coordenadas xy) e o cálculo é realizado a partir de um valor médio representativo:

$$
\overline{\mathrm{z}}=\frac{\sum_{1}^{\mathrm{n}} \mathrm{y}_{\mathrm{i}}}{\mathrm{n}_{\mathrm{i}}}
$$

No procedimento para a determinação do valor de $\overline{\mathrm{Z}}$ recomenda-se a tomada de uma fotografia da árvore, de modo a se obter sua silhueta. é evidente que neste processo se deve cuidar para que uma escala seja incluída na fotografia, procedimento este também sugerido pelo fabricante do LI-2000 para que se obtenha a distância percorrida pela luz dentro da copa em cinco ângulos pré-estabelecidos; para exemplificar os cálculos de como o sensor do luxímetro é sensível à radiação proveniente de todos os pontos da copa, pode-se optar por seccionar a copa em ângulos de $5^{\circ}$ (36 pontos) e, para cada um deles, calcular o valor de $\mathrm{z}_{\mathrm{i}}$, como mostra a Figura 1. A forma de cálculo de $\bar{z}$ é uma simplificação, já que a radiação adentra a copa por um número infinito de pontos tornando-se evidente que quanto maior o número de amostras melhor será a estimativa de $\overline{\mathrm{z}}$ que, no presente exemplo, é dada por:

$$
\bar{z}=\frac{\sum_{i=0}^{180} z_{i}}{36}
$$

\section{Descrição do luxímetro utilizado}

As medidas de $\mathrm{T}(\alpha)$ foram realizadas com um luxímetro digital da ICEL com as seguintes especificações: para a escala de 0 a 2000 lux a resolução foi de 1 lux, com precisão nominal de 2\%; para a escala de 2000 a 20000 lux a resolução foi de 10 lux e a precisão de $\pm 2 \%$; para a escala de 20000 a 50000 lux a resolução foi de 100 lux e a precisão de $\pm 5 \%$.

Ao sensor do luxímetro foi acoplado um sistema de filtro constituído de um anel de suporte ao qual foi fixado um disco de plástico azul. A avaliação das características óticas do filtro foi realizada procedendo-se a uma série de medidas com e sem o filtro e se relacionando estas medidas, obtendo-se um valor médio de $30 \%$, ou seja:

$$
\frac{\int_{400}^{490} E \lambda d \lambda}{\int_{400}^{700} E \lambda d \lambda} \times 100 \equiv 30 \%
$$

sendo $E \lambda$ a energia luminosa associada ao comprimento de onda $\lambda$ (mícron).

Como em média no espectro solar a fração espectral entre 400 e $490 \mathrm{~nm}$ (azul e violeta) corresponde a cerca de 32\% da faixa visível (Robinson, 1966), assumiu-se que o filtro se mostrou eficiente para o objetivo de permitir a incidência sobre o sensor do luxímetro a radiação dentro dessa banda.

\section{RESULTADOS E DISCUSSÃO}

\section{Determinação de área foliar em limeiras ácidas 'Tahiti'}

As regressões lineares necessárias para aplicabilidade dos métodos indiretos envolvendo medidas das dimensões das folhas e de sua massa, apresentaram coeficientes de determinação elevados $\left(r^{2}>0,99\right)$, evidenciando excelente ajuste entre as variáveis. $\mathrm{O}$ valor do coeficiente $\mathrm{K}$ para folhas de lima ácida na regressão linear $\mathrm{AF}=\mathrm{K}\left(\mathrm{C}^{*} \mathrm{~L}\right)$ foi de 0,72 , resultado próximo ao obtido por Angelocci \& Valancogne (1993) para macieira e para fruteiras em geral apresentando folhas com formato aproximadamente elipsoidal. A relação AF/MS obtida foi de $90,72 \mathrm{~cm}^{2} \mathrm{~g}^{-1}$.

Os resultados referentes à comparação entre os métodos indiretos testados em três plantas avaliadas, estão resumidos na Tabela 1. Para a planta 1 foram comparados os métodos indiretos envolvendo medidas de massa seca e de dimensões foliares e os baseados em transmissão de luz. No primeiro grupo de métodos as diferenças foram mínimas, 2,68\% entre valor máximo dado pelo método de matéria seca (MSAF) e mínimo pela contagem de folhas $(\mathrm{CF})$. Embora sejam métodos indiretos, 
Tabela 1. Área foliar de três limeiras ácidas 'Tahiti' $\left(\mathrm{m}^{2}\right)$, determinada pelos métodos indiretos IAF-LUX e LAI-2000, analisadas por quadrante (Q1 a Q4) e em duas direções (DIR)

\begin{tabular}{|c|c|c|c|c|c|c|c|c|c|c|c|c|}
\hline \multirow{3}{*}{ Método } & \multicolumn{6}{|c|}{ Planta 1} & \multicolumn{3}{|c|}{ Planta 2} & \multicolumn{3}{|c|}{ Planta 3} \\
\hline & Q1 & Q2 & Q3 & Q4 & Total & \multirow{2}{*}{$\begin{array}{c}\text { DIF** } \\
(\%)\end{array}$} & DIR1 & DIR2 & TOT & DIR1 & DIR2 & TOT \\
\hline & & & $\left(m^{2}\right)$ & & & & \multicolumn{6}{|c|}{$\left(m^{2}\right)$} \\
\hline I & 4067 & 3597 & 367 & 4721 & 1605 & +810 & 42,64 & 44,78 & $87,4^{(*)}$ & 27,52 & 31,94 & 59,50 \\
\hline LAI $\angle O O O$ & $4,0 /$ & 35,91 & 30,1 & $4 l, \angle I$ & 100,5 & $+8,10$ & 23,96 & 27,18 & 51,1 & - & - & - \\
\hline IAF-LUX & 41,96 & 40,85 & 36,71 & 39,09 & 158,6 & $+6,80$ & 22,00 & 21,35 & 41,30 & 32,69 & 36,04 & 68,73 \\
\hline MSAF & 36,16 & 42,38 & 38,43 & 32,21 & 149,2 & $+0,47$ & & - & - & - & - & - \\
\hline $\mathrm{CF}$ & 44,62 & 37,49 & 41,45 & 21,35 & 145,2 & $-2,22$ & - & - & - & - & - & - \\
\hline $\mathrm{CF}^{*}$ & 34,55 & 39,92 & 39,07 & 33,71 & 147,2 & $-0,88$ & - & - & - & - & - & - \\
\hline $\mathrm{DL}$ & 44,65 & 37,52 & 41,78 & 21,37 & 148,5 & 0,00 & 18,81 & 16,0543 & $34,9^{(-)}$ & - & - & - \\
\hline
\end{tabular}

Cada valor corresponde à média de 16 leituras do LAI-2000 em um ponto de medida ( $Q$ ou DIR) e de 8 leituras (IAF-LUX) em quatro pontos diferentes (Q ou DIR)

* 0 cálculo de área foliar total foi realizado com uso de um valor médio de área foliar amostrado individualmente para cada quadrante analisado. 0 cálculo no método CF foi a partir da média geral da área foliar individual de todos os quadrantes

** Diferença percentual das metodologias com relação ao valor obtido com DL

assumiu-se que qualquer um deles poderia ser tomado como a referência de comparação para definir o desempenho dos métodos com base em transmissão de luz. O método das medidas de dimensões lineares (DL) foi adotado nas comparações, por ter sido usado nas plantas 1 e 2 .

Para a árvore 1 constata-se que os métodos com base na transmissão de luz no dossel superestimaram os indiretos com uso de dimensões das folhas e massa seca. As superestimativas foram ligeiramente superiores ao se utilizar o LAI-2000 $(+8,1 \%$ ou $\left.+12,0 \mathrm{~m}^{2}\right)$ contra $+6,80 \%\left(+10,1 \mathrm{~m}^{2}\right)$ do IAF-LUX em comparação a DL. Esses desvios podem ser considerados pequenos em relação à área foliar total da planta. Com base na afirmativa de Welles \& Cohen (1996) de que os métodos nos quais se usa o princípio da transmissão de luz testados ao longo de vários anos apresentam, em média, acurácia de $20 \%$, com exceção para as culturas que não satisfazem as hipóteses randômicas de distribuição de folhas do modelo, quando os erros se tornam expressivos e podem superar $40 \%$ (Norman \& Welles, 1991); pode-se afirmar, então, que os resultados obtidos com os métodos indiretos no presente trabalho estão coerentes com a base teórica e se mostram aplicáveis em estimativas de AF em plantas isoladas de lima ácida 'Tahiti'.

Com os resultados de AF por quadrante da planta 1, houve tendência do IAF-LUX apresentar valores superiores ao LAI2000 (Tabela 1, Figura 2A). Previa-se uma suscetibilidade maior do IAF-LUX aos erros visto que o luxímetro não possui o aparato para limitar visadas (no caso $90^{\circ}$ ) nem direcionamento em ângulos específicos (sensor "olho de peixe") como o LAI2000; entretanto, os resultados das medidas do LAI-2000 não evidenciaram ganhos em comparação ao IAF-LUX, que se aproximou mais do método de referência (Figura 2A). A suscetibilidade dos métodos a essa fonte de erro (proximidade de plantas vizinhas, de troncos e de caules) evidencia a importância da escolha do local de posicionamento do sensor para a realização das leituras.

Apenas no quadrante $4 \mathrm{o}$ IAF-LUX não superou o LAI2000 , resultado que contribuiu para aumentar o erro (total) no valor total de AF estimado com o LAI-2000. A diferença entre os valores obtidos com o MSAF e os obtidos com o LAI-2000 neste quadrante foi de $15 \mathrm{~m}^{2}$, o que também foi verificado para o IAF-LUX em menores proporções $\left(7 \mathrm{~m}^{2}\right)$. Esta maior suscetibilidade ao erro do LAI-2000 no referido quadrante, pode
A.

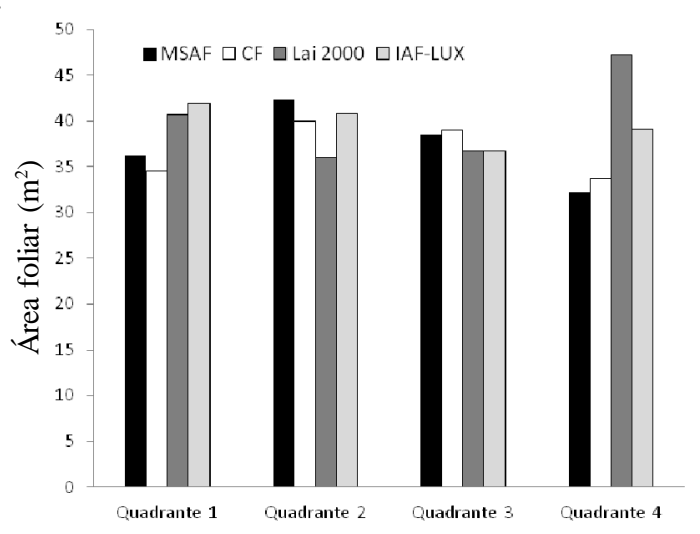

B.

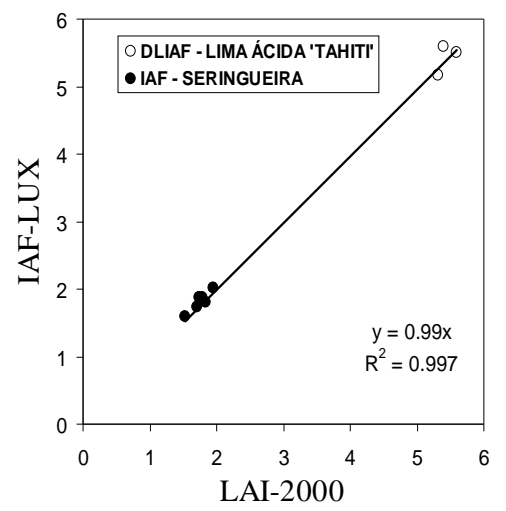

Figura 2. Estimativa de área foliar $\left(\mathrm{m}^{2}\right)$ utilizando-se técnicas com uso de massa seca (MSAF) e dimensões da folha (CF) e técnicas de medida de transmissão da radiação pelo dossel, analisando-se separadamente cada quadrante da planta 1 de lima ácida (A); relação entre os valores de IAF em seringueira obtidos com o método proposto (IAFLUX) e com o equipamento comercial (LAI-2000) e do DLIAF em três plantas de lima ácida "Tahiti", medidas realizadas conforme Planta 1 da Tabela 2 (B)

estar relacionada à interferência da planta vizinha já que a direção da visada realizada no $\mathrm{Q}_{4}$ foi feita na linha de plantio, que possuía espaçamento de $7 \mathrm{~m}$. Verificaram-se subestimativas nos quadrantes Q1 e Q4 quando as leituras foram direcionadas para entrelinha e superestimativas para o direcionamento nas linhs Q2 e Q3 (Tabela 1), indicando que os erros foram 
compensados nos dois métodos. Se a análise fosse realizada desconsiderando-se o $\mathrm{Q}_{4}$ o valor de AF para o IAF-LUX seria de $159 \mathrm{~m}^{2}$, não alterando muito o resultado encontrado mas, empregando-se o LAI-2000, este valor passaria a ser $151 \mathrm{~m}^{2}$, muito próximo do MSAF cujo resultado indica que, além da minimização dos erros com recursos disponíveis pelo aparelho (Li-Cor, 1992), o posicionamento em relação à planta vizinha pode interferir muito no bom desempenho, ao se utilizar este aparelho. Como cada estudo tem sua particularidade, o número de medidas por planta e o posicionamento das visadas devem mudar, dependendo da cultura e do espaçamento entre plantas, quando o objetivo for a determinação de AF de plantas isoladamente.

Com relação à planta 2 (Tabela 1), as medidas com LAI-2000 foram realizadas em duas etapas (antes e após a retirada de folhas) para avaliação da sensibilidade do instrumento a possíveis variações na quantidade de folhas. O procedimento utilizado na retirada com medida das dimensões foliares de subamostras e contagem de folhas, foi criterioso. A remoção foi uniforme em toda a planta evitando o aparecimento de regiões sem folhas que pudessem violar as hipóteses do modelo (LAI-2000). Para tanto, dividiu-se a planta em quadrantes e em cada um foram coletadas aproximadamente 3900 folhas com pequenas flutuações em torno deste valor. Foram determinados $\mathrm{C}$ e L em aproximadamente $3 \%$ do total das folhas coletadas, para posterior aplicação do método DL. Os resultados encontrados para o LAI-2000 nessa planta indicam que tal instrumento detecta muito bem as variações temporais da área foliar total na árvore. Verifica-se que a diferença entre os valores de $\mathrm{AF}$ antes e depois da poda é de $36,3 \mathrm{~m}^{2}$, bem próximo do valor $34.8 \mathrm{~m}^{2}$ encontrado no DL.

O valor absoluto total de AF encontrado com o LAI-2000 após a remoção das folhas da planta 2 foi bem diferente $(+46$ $\mathrm{m}^{2}$ ) dos valores observados nas medidas com a técnica DL. O IAF-LUX teve melhor desempenho (+18\%), sendo que os dois métodos mantiveram a tendência de superestimativa. Essas diferenças observadas podem estar ligadas, como verificado para a planta 1, ao local de realização das leituras na linha de plantio, possibilitando interferência da planta vizinha, com maior susceptibilidade ao erro novamente para o LAI-2000.

Comparando os valores de AF total das plantas estimados com os dois métodos indiretos, verifica-se uma proximidade maior dos valores observados na planta 1 , com diferença de aproximadamente $2 \mathrm{~m}^{2}$ (Tabela 1). Para as duas outras plantas a diferença ficou na ordem de $10 \mathrm{~m}^{2}$. Tais resultados podem ser atribuídos ao único procedimento diferente utilizado nessas plantas em relação à planta 1 , que foi a divisão da planta em duas partes, utilizando-se um limitador de visada de $180^{\circ}$ (Plantas 2 e 3; Figura 1A), reforçando mais uma vez a importância da amostragem nesse tipo de análise e sugerindo que, quanto maior o número de pontos medidos melhor também será a resposta, em que o ideal seria trabalhar, sempre, com o limitador de visada de 300 e analisar separadamente 12 partes $(\varphi / 12)$. Apesar das diferenças encontradas ao se comparar os resultados dos métodos em cada um dos 4 quadrantes, os erros foram compensados e os valores finais ficaram muito próximos dos métodos diretos.

A variabilidade das leituras do IAF-LUX entre quadrantes é menor que a do LAI-2000 e pode estar ligada ao sistema de cálculo no qual são utilizados valores médios de transmitância obtidos em 4 pontos do quadrante (Figura 1A). A área foliar para cada quadrante foi modificada também em função de $\bar{z}$ e $\varphi$ variáveis em cada um. Essas duas variáveis de entrada do modelo IAF-LUX são as que mais interferem nos resultados da área foliar total, pois são termos multiplicativos da Eq. 9. É importante salientar que para oLAI-2000 é calculada, primeiro, a densidade foliar, utilizando-se as coordenadas xy e os valores de $\mu$ variam, dependendo de $\phi$. Se o interesse for estudar $\mu$, a obtenção de valores médios confiáveis dependerá de uma boa amostragem e detalhamento de perfis da árvore analisada. Verifica-se que os valores de $\mu$ se aproximaram de 2 em todas as árvores avaliadas e foram inferiores para as plantas apresentando maior AF (Tabela 2). Os valores de $\mu$ referentes ao IAF-LUX foram obtidos dividindo-se a área foliar encontrada (Eq. 9) pelo volume de copa.

As dificuldades de uma amostragem direta de AF são limitantes quando se trabalha com árvores e ainda mais críticas se o objetivo for a obtenção de um valor médio representativo de um pomar. Neste caso, quantas medidas (plantas) são necessárias e quantas serão possíveis? A solução, mediante o uso de metodologias alternativas, também apresenta problemas; como exemplo, nos trabalhos de Bignami \& Rossini (1996), Coelho Filho et al. (2005) e Marcon et al. (2011), nos quais os autores estimaram AF com base em silhuetas das

Tabela 2. Variáveis de entrada dos modelos $(\phi, \varphi$ e $\bar{z})$ e os valores de $\mu$ e DLIAF de três árvores de lima ácida 'Tahiti' estimados pelas técnicas IAF-LUX e LAI 2000

\begin{tabular}{|c|c|c|c|c|c|c|c|c|c|c|c|}
\hline \multirow{2}{*}{ VAR } & \multicolumn{5}{|c|}{ Planta 1} & \multicolumn{3}{|c|}{ Planta 2} & \multicolumn{3}{|c|}{ Planta 3} \\
\hline & Q1 & Q2 & Q3 & Q4 & MÉDIA & DIR1 & DIR2 & MÉDIA & DIR1 & DIR2 & MÉDIA \\
\hline$\mu$-Lai2000 & 1,51 & 1,98 & 1,84 & 2,24 & 1,89 & $\begin{array}{l}2,18^{* *} \\
2,00\end{array}$ & $\begin{array}{l}2,40 \\
2,45\end{array}$ & $\begin{array}{l}2,29 \\
2,23\end{array}$ & 1,77 & 2,20 & 1,98 \\
\hline$\mu$-IAF-Lux & 1,73 & 2,38 & 1,98 & 1,33 & 1,85 & 1,84 & 1,92 & 1,88 & 2,23 & 2,48 & 2,35 \\
\hline$\phi^{*}$ & 24,29 & 17,16 & 18,54 & 29,21 & 22,30 & $\begin{array}{l}19,56 \\
11,98\end{array}$ & $\begin{array}{l}18,66 \\
11,07\end{array}$ & $\begin{array}{l}19,11 \\
11,52\end{array}$ & 15,55 & 14,52 & 15,03 \\
\hline$\varphi$ & 6,98 & 8,38 & 8,28 & 7,18 & 7,71 & $\begin{array}{l}9,35^{\star \star} \\
5,85\end{array}$ & $\begin{array}{l}8,53 \\
5,43\end{array}$ & $\begin{array}{l}8,93 \\
5,64\end{array}$ & 6,09 & 6,66 & 6,37 \\
\hline $\bar{z}$ & 2,00 & 1,62 & 1,47 & 1,79 & 1,72 & 1,44 & 1,66 & 1,55 & 1,60 & 1,60 & 1,60 \\
\hline DLIAF LAI & 5,91 & 4,31 & 4,65 & 6,42 & 5,32 & $\begin{array}{l}4,56 \\
4,09\end{array}$ & $\begin{array}{l}5,25 \\
5,00\end{array}$ & $\begin{array}{l}4,91 \\
4,55\end{array}$ & 4,52 & 4,80 & 4,66 \\
\hline DLIAF LUX & 6,00 & 4,87 & 4,43 & 5,38 & 5,17 & 3,76 & 3,93 & 3,84 & 5,59 & 5,41 & 5,50 \\
\hline
\end{tabular}

* Os valores de $\phi$ e $\varphi$ utilizados são os mesmos para as duas metodologias, sendo que o primeiro foi calculado com uso do programa C2000

** Planta antes da remoção das folhas para análise 
plantas, assumindo que a densidade de folhas é constante e em trabalhos com macieira, cujas estimativas foram feitas por meio das relações com parâmetros vegetativos, como o diâmetro ou perímetro do tronco ou de ramos, aplicados (Holland, 1968; Palmer, 1987; Angelocci \& Valancogne, 1993), quando as relações são variáveis no tempo em função do manejo da cultura, como as podas. Por isto e apesar do pequeno número de repetições no presente estudo, conclui-se que o método IAF-LUX pode ser empregado para medidas de IAF em árvores isoladas mesmo sob condições de elevada densidade de folhagem, como na Planta 1.

\section{Determinação de IAF em seringueira}

As estimativas de IAF feitas pelo método proposto IAFLUX no seringal resultaram em valores concordantes com aquelas obtidos com o (LAI-2000). Nas seis repetições realizadas o desvio em relação ao LAI-2000 ficou entre -7,4\% e $+1,6 \%$, indicando uma leve tendência de subestimativa por parte do método proposto. Apesar da faixa de valores amostrados no seringal ter ficado entre 1,59 e 2,01, pode-se notar o bom desempenho do IAF-LUX para esta condição (Tabela 3).

Tabela 3. Valores de IAF obtidos com o método proposto (IAF - LUX) e com o LAI-2000 com os respectivos erros, em seringal

\begin{tabular}{|c|c|c|c|}
\hline \multirow{2}{*}{ Posição } & IAF - LUX & LAI - 2000 & \multirow{2}{*}{$\begin{array}{c}\frac{\text { IAFLM-LAI2000 }}{\text { LAI2000 }} \times 100 \\
(\%)\end{array}$} \\
\hline & \multicolumn{2}{|c|}{$\left(m^{2} m^{-2}\right)$} & \\
\hline 1 & 1,70 & 1,73 & $-1,7$ \\
\hline 2 & 1,95 & 2,01 & $-2,9$ \\
\hline 3 & 1,74 & 1,87 & $-7,4$ \\
\hline 4 & 1,53 & 1,59 & $-3,9$ \\
\hline 5 & 1,79 & 1,87 & $-4,4$ \\
\hline 6 & 1,83 & 1,80 & $+1,6$ \\
\hline
\end{tabular}

Analisando os valores obtidos de IAF e DLIAF no seringal e em três plantas de lima ácida 'Tahiti' com os dois equipamentos (IAF-LUX e LAI-2000), é interessante notar que a relação encontrada entre as medidas se adequam perfeitamente à mesma equação de regressão em ambos os conjuntos de dados. Isto permite inferir que na faixa de abrangência das medidas apresentadas se espera bom desempenho do método IAF-LUX para estimativa do IAF, tanto para culturas com cobertura contínua do terreno como para árvores isoladas. O coeficiente de correlação de 0,99 reflete a excelente concordância entre os métodos (Figura 2B).

Considerando-se que o LAI-2000 é uma metodologia tomada como referência em diversos estudos em que se verificam baixas correlações (Monte et al., 2007; Chason et al., 1991; Macfarlanre et al., 2000) e erros bem superiores aos obtidos no presente estudo decorrentes do tipo de cobertura que não satisfaz as premissas necessárias para o uso da Eq. 1 (Welles, 1990; Rich, 1990; Macfarlane et al., 2000) ou quando o equipamento é utilizado inapropriadamente, a técnica ora proposta apresentase como boa alternativa, pois tem as vantagens do baixo custo do sensor usado em relação ao LAI-2000 e de não necessitar de softwares específicos para análise dos dados.

\section{ConclusõES}

1. Os dois métodos (LAI 2000 e IAF-LUX) com uso de transmissão de radiação através do dossel, apresentaram boa concordância com a determinação a partir de dimensões ou de massa seca das folhas.

2. O LAI-2000 detectou muito bem as variações temporais da área foliar total de árvore de lima ácida 'Tahiti' .

3. Embora seja necessário realizar um número maior de comparações com métodos-padrão para análise das limitações em diferentes condições de trabalho, conclui-se que a determinação com a técnica aqui denominada IAF-LUX, é potencialmente promissora na estimativa de área foliar de dosséis quando não se exige um nível de precisão muito elevado.

\section{LITERATURA CITADA}

Allen, R. G.; Pereira, L. S.; Raes, D.; Smith, M. Crop evapotranspiration: Guidelines for computing crop water requirements. Rome: FAO, 1998, 297p. FAO. Irrigation and Drainage Paper, 56

Angelocci, L. R.; Valancogne, C. Leaf area and water flux in apple trees. Journal of Horticultural Science, v.67, p.299307, 1993.

Arias, D; Calvo-Alvarado, J; Dohrenbusch, A. Calibration of LAI-2000 to estimate leaf area index (LAI) and assessment of its relationship with stand productivity in six native and introduced tree species in Costa Rica. Forest Ecology and Management, v.247, p.185-193, 2007.

Barclay, H. J.; Trofmow, J. A. Relationship of readings from the LICOR canopy analyzer to total one-sided leaf area index and stand structure in immature Douglas-fir. Forest Ecology and Management, v.132, p.121-126, 2000.

Bignami, C.; Rossini, F. Image analysis of leaf area index and plant size of young hazelnult plants. Journal of Horticultural Science, v.71, p.113-121, 1996.

Brantley, S. T.; Zinnert, J. C;Young, D. R. Application of hyperspectral vegetation indices to detect variations in high leaf area index temperate shrub thicket canopies. Remote Sensing of Environment, v.115, p.514-523, 2011.

Chason, J. W.; Baldocchi, D. D.; Huston, M. A. A comparison of direct and indirect methods for estimating forest canopy leaf area.Agricultural and Forest Meteorology, v.57, p.107128, 1991.

Coelho Filho, M. A.; Angelocci, L. R.; Campeche, L. F. S. M. ; Rojas, J. S. D. ; Folegatti, M. V. Relações entre transpiração máxima, área foliar e evapotranspiração de referência em pomar jovem de lima ácida 'Tahiti'(Citrus latifolia Tan.). Revista Brasileira de Agrometeorologia, v.12, p. 265-274,2004.

Coelho Filho, M. A.; Angelocci, L. R.; Vasconcelos, M. R. B.; Coelho, E. F. Estimativa da área foliar de plantas de lima ácida 'Tahiti' usando métodos não-destrutivos. Revista Brasileira de Fruticultura, v.27, p.163-167, 2005. 
Coelho Filho, M. A.;Coelho, E. F.; Cruz, L. L. Uso da transpiração máxima de mamoeiro para o manejo de irrigação por gotejamento em regiões úmidas e sub-úmidas. Cruz das Almas: Embrapa Mandioca e Fruticultura Tropical, 2007.43p. Documento 162

Cutini, A.; Matteucci, G.; Mugnozza, G. S. Estimation of leaf area index with the Li-Cor LAI 2000 in deciduous forests. Forest Ecology and Management, v.105, p.55-65, 1998.

Demarez, V.; Duthoit, S.; Beret, F.; Weiss, M. ;Dedieu, G. Estimation of leaf area and clumping indexes of crops with hemispherical photographs. Agricultural and Forest Meteorology, v.148, p.644-655, 2008.

Fassnacht, K. S.; Gower, S. T.; Norman, J. M.; McMurtrie, R. E. A comparison of optical and direct-methods for estimating foliage surface-area index in forests. Agricultural and Forest Meteorology, v.71, p.183-207, 1994.

Fuentes, S.; Palmer, A. R.; Taylor, D.; Zeppel, M.; Whitley, R.; An automated procedurefor estimating the leaf area index (LAI) of woodland ecosystems using digital imagery, MATLAB programming and its application to an examination of the relationship between remotely sensed and field measurements of LAI. Functional Plant Biology, v. 35, p.10701079, 2008.

Herbert, D. A.; Fownes, J. H. Effects of leaf aggregation in a broad-leaf canopy on estimates of leaf area index by the gap-fraction method.Forest Ecology and Management, v.97, p.277-282, 1997.

Holland, D. A. The estimation of total leaf area on tree.Annual Report of the East Malling Research Station for 1967, p.101107, 1968.

Hollinger, D. Y. Leaf and simulated whole-canopy photosynthesis in two co-occurring tree species Ecology, v.73, p.1-14, 1992.

Kucharik, C. J.; Norman, J. M. ; Grower, S. T. Measurement of branch area and adjusting leaf area index indirect measurement. Agriculture Forest Meteorology, v.91, p.69$88,1998$.

Lang, A. R. G.; McMurtrie, R. E.; Benson, M. L. Validity of surface area indices of Pinus radiata estimated from transmittance of the sun's beam. Agricultural and Forest Meteorology, v.57, p.157-170, 1991.

Lang, A. R. G.; Yenqin, X.; Norman, J. M. Crop structure and the penetration of direct sunlight. Agricultural and Forest Meteorology, v.35, p.83-101, 1985.

Lara, M. A. S.; Pedreira, C. G. S.Estimativa da assimilação potencial de carbon emdosséis de espécies de braquiária. Pesquisa Agropecuária Brasileira, v.46, p.743-750, 2011.

Leblanc, S. G.; Chen, J. M.; Fernandes, R.; Deering, D. W.; Conley, A. Methodology comparison for canopy structure parameters extraction for hemispherical photographs in boreal forests.Agricultural and Forest Meteorology, v.129, p.187-207, 2005.

Leong, W.; Lemeur, R.; Yoon, P. K. Characterization of leaf area index and canopy light penetration of Heveabrasiliensis by hemispherical photography.Journal of the Rubber Research Institute of Malaya, v.30, p.80-90, 1982.

Li-Cor. LAI 2000 plant canoyanalyser: Instruction manual. Li-Cor Inc. Lincoln, Nebraska. 1992. 182p.
Linhares, C. A.; Ponzoni, F. J.; Shimabukuro, Y. E. Relação entre volume de madeira e Índice de Área Foliar em um povoamento de Pinus spp.: Estudo de caso. Revista Árvore, v.24, p.47-54, 2000.

Macfarlane, C.; Coote, M.; White, D. A.; Adams, M. A. Pothograph exposure affects indirect estimation of leaf area in plantations of Eucalyptus globulusLabill. Agricultural and Forest Meteorology, v.100, p.155-168, 2000.

Macfarlane, C.; Hoffman, M.; Eamus, D.; Kerp, N.; Higginson, S.; McMurtrie, R.; Adams, M. Estimation of leaf area index in eucalypt forest using digital photography. Agricultural and Forest Meteorology, v.143, p.176-188, 2007.

Majasalmi, T; Rautiainen, M.; Stenberg, P.; Rita, H. Optimizing the sampling scheme for LAI-2000 measurements in a boreal forest. Agricultural and Forest Meteorology, v.154- 155, p.3843, 2012.

Marcon, M.; Mariano, K.; Braga, R. A.; Paglis, C. M.; Scalco, M. S.; Estimation of total leaf area in perennial plants using image analysis Horgan, G. W. Revista Brasileira de Engenharia Agricola e Ambiental, v.15, p.96-101, 2011.

Miller, J.B. A formula for average foliage density. Austalia Journal of Botany, v.15, p.141-144, 1967.

Monte, M. A.; Reis, M. G. F.; Reis, G. G.; Leite, H. G. L.; Stocks, J. J. Métodos indiretos de estimação da cobertura de dossel em povoamentos de clone de eucalipto. Pesquisa Agropecuária Brasileira, v.42, p.769-775, 2007.

Norman, J. M.; Campbell, G. S. Canopy structure. In: Pearcy, R. W.; Ehleringer, J.; Mooney, H. A.; Rundel, P. W. (eds). Plant physiological ecology: Field methods and instrumentation. London: Chapman and Hall, 1989. p.301-326.

Palmer, J. W. The measurements of leaf area in apple trees. Journal of Horticultural Science, v.62, p.5-10, 1987.

Paula, S. A.; Lemos Filho, J. P. Dinâmica do dossel em mata semidecídua no perímetro urbano de Belo Horizonte, MG. Revista Brasileira de Botânica, v.24, p.545-551, 2001.

Rich, P. M. Characterizing plant canopies with hemispherical photograpry. Remote Sensing Reviews, v.5, p.13-18, 1990.

Righi, C. A.; Bernardes, M. S. The potential for increasing rubber production by matching tapping intensity to leaf area index. Agroforestry Systems, v.72, p.1-13, 2008.

Righi, C. A.; Luns, A. M. P.; Bernardes, M. S.; Pereira, C. R.; Teramoto, E. R.; Favarin, J. L. Coffee water use in agroforestry system with rubber trees. Revista Árvore, v.32, p.781-792, 2008.

Robinson, N. Solar radiation. Amsterdam, New York. Elsevier Publishing Company. 1966. 347p.

Sbrissia, A. F.; Silva, S. C. Comparação de três métodos para estimativa do índice de área foliar em pastos de capimmarandu sob lotação contínua. Revista Brasileira de Zootecnia, v.37, p.212-220, 2008.

Schlerf, M.; Atzberger, C.; Hill, J. Remote sensing of forest biophysical variables using HyMap imaging spectrometer data. Remote Sensing of Environment, v.95, p.177-194, 2005.

Silva, T. G. F. da; Moura, M. S. B. de; Zolnier, S.; Soares, J. M.; Souza, L. S. B. de; Brandão, E. O. Variação do balanço de radiação e de energia da cana-de-açúcarirrigada no semiáridobrasileiro. Revista Brasileira de Engenharia Agrícola e Ambiental, v.15, p.139-147, 2011. 
Smethurst, P. J.; Baillie, C.; Cherry, M.; Holz, G. Fertilizer effects on LAI and growth of four Eucalyptus nitens plantations. Forest Ecology and Management. v. 176, p. 531-42, 2003.

Sommer, K. J.; Lang, A. R. G. Comparative analysis of two indirect methods of measuring leaf area index as applied to minimal and spur pruned grape vines. Australia Journal of Plant Physiology, v.21, p.197-206, 1994.

Stenberg, P. Correcting LAI-2000 estimates for the clumping of needles in shoots of conifers. Agricultural and Forest Meteorology, v.79, p.1-8, 1996.

Stroppiana, D.; Boschetti, M.; Confalonieri, R.; Stefano, B.; Alessandro, B. P. Evaluation of LAI-2000 for leaf area index moni-toring in paddy rice. Field Crops Research, v.99, p.167170, 2006.

Villalobos, F. J.; Orgaz, F.; Mateos, L. Non-destructive measurement of leaf area on olive (Oleaeuropaea L.) trees using a gap inversion method. Agricultural and Forest Meterology, v.73, p.29-42, 1995.
Welles, J. M. Some indirect methods of estimating canopy structure. Remote Sensing Reviews, v.5, p.31-43, 1990.

Welles, J. M.; Cohen, S. Canopy structure measurement by gap fraction analysis using commercial instrumentation. Journal of Experimental Botany, v.47, p.1335-1342, 1996.

Welles, J. M; Norman, J. M. Instrument for indirect measurement of canopy architecture. Agronomy Journal, v.83, p.818-825, 1991.

Xavier, A. C.; Soares, J. V.; Almeida, A. C. de. Variação do índice de área foliar em clones de eucalipto ao longo de seu ciclo de crescimento. Revista Árvore, v.26, p.421-427, 2002.

Xavier, A. C.; Vettorazzi, C. A. Leaf area index of ground covers in a subtropical watershed. Scientia Agricola, v.60, p.425431, 2003.

Zhang, Y.; Chen, J. M.; Miller, J.r.; Determining digital hemispherical photograph exposure for leaf area index estimation. Agricultural and Forest Meteorology, v.133, p.166-181, 2005. 